\title{
Pacific
}

Journal of

Mathematics

\section{STATIONARY SURFACES IN MINKOWSKI SPACES. I. A REPRESENTATION FORMULA}

\author{
JIANGFAN LI
}




\title{
STATIONARY SURFACES IN MINKOWSKI SPACES, I. A REPRESENTATION FORMULA
}

\author{
JIANGFAN LI
}

\begin{abstract}
Gu has derived a representation formula for stationary surfaces in the 3-dimensional Minkowski space using the Legendre transformation. By a different method, we generalize his results to any Minkowski spaces.
\end{abstract}

1. Introduction. Given a differentiable map $f:\left(M_{1}, g_{1}\right) \rightarrow\left(M_{2}, g_{2}\right)$ between two manifolds with nondegenerate (definite or indefinite) metrics, its energy is defined as

$$
E(f)=\int_{M_{1}}|d f|^{2} d \text { vol }_{g_{1}} .
$$

A critical point of this energy functional is called a harmonic map. Let $\left(M_{2}, g_{2}\right)$ be any Minkowski space, $\left(M_{1}, g_{1}\right)$ an immersed submanifold of $M_{2}$ such that the induced metric $g_{1}$ is nondegenerate except possibly on a subset of codimension at least one. Let $f: M_{1} \rightarrow M_{2}$ be the inclusion map. Then its energy is still well defined. If $f$ is a critical point for the energy (i.e., the mean curvature vanishes), $M_{1}$ is called a stationary submanifold in the Minkowski space $M_{2}$; if $M_{1}$ has real dimension two, then we call it a stationary surface, since in general it is not extremal-neither minimal nor maximal. Although minimal surface theory in Euclidean spaces has been studied for hundreds of years, the analogous stationary surface theory in Minkowski spaces only has a very short history. Suppose $p$ is a point on the surface $S$ in a Minkowski space. We call $p$ an elliptic, hyperbolic, parabolic point if the induced metric on $S$ at $p$ is definite, indefinite but nondegenerate, degenerate respectively. A powerful tool to study the stationary surface is to use isothermal coordinates, whose existence is well-known. At a hyperbolic point, if the metric is written as $d s^{2}=f(x, y) d x d y$, then $(x, y)$ are called characteristic coordinates. This paper is inspired by Gu's work [1] and [2], of which many ideas are carried on here.

2. Representation formula. We denote by $\mathbb{R}^{m, n}$ the Minkowski space of signature $(m, n)$, i.e., the vector space $R^{m+n}$ with the metric 


$$
d s^{2}=\left(\sum_{i=1}^{m}-\sum_{i=m+1}^{m+n}\right) d x_{i}^{2} .
$$

Let

$$
\begin{aligned}
K^{m, n} & =\left\{x \in \mathbb{R}^{m, n} \mid\langle x, x\rangle=0\right\} \\
\mathbb{N}^{m, n} & =\left\{\left(\zeta_{1}, \ldots, \zeta_{m+n}\right) \in \mathbb{C}^{m+n} \mid\left(\sum_{i=1}^{m}-\sum_{i=m+1}^{m+n}\right) \zeta_{i}^{2}=0\right\}
\end{aligned}
$$

be the real and complex null cones. We will see that stationary surfaces are equivalent to maps into null cones.
A. Elliptic stationary surfaces.

Theorem 2.1. Suppose $X: S \rightarrow \mathbb{R}^{m, n}$ is an elliptic stationary surface, and $p \in S$. Then there exists a neighborhood $U$ of $p$, and isothermal coordinates $(x, y)$ on $U$, such that $X$ can be written

$$
X(\zeta)=X(0)+\operatorname{Re} \int_{0}^{\zeta} \Phi(\zeta) d \zeta \quad(\zeta=x+i y)
$$

where

$$
\Phi: U \rightarrow \mathbb{N}^{m, n} \subset \mathbb{C}^{m+n}
$$

is holomorphic, satisfying

$$
\|\Phi\|_{m, n}^{2} \neq 0 \text { everywhere. }
$$

Conversely, any holomorphic map (2) satisfying (3) gives rise to an elliptic stationary surface (1) in $\mathbb{R}^{m, n}$.

Proof. Suppose $X: S \rightarrow \mathbb{R}^{m, n}$ is an elliptic immersion. Then under the isothermal coordinates $(x, y)$ on $S$, the map $X$ is stationary iff

$$
X_{x x}+X_{y y}=0 \text {. }
$$

Let

$$
\zeta=x+i y, \quad \bar{\zeta}=x-i y
$$

Then

$$
\Phi=X_{\zeta}=\frac{1}{2}\left(X_{x}-X_{y}\right)
$$

satisfies

$$
\Phi_{\bar{\zeta}}=0
$$


i.e., $\Phi$ is holomorphic Since $(x, y)$ are isothermal, the image of $\Phi$ lies in $\mathbb{N}^{m, n}$. Since $X$ is an immersion, (3) holds. Conversely, it is straightforward to derive (4) from (1), (2) and (3).

Theorem 2.1 can be stated as the following multi-tuplets phenomena (as $\mathrm{Gu}$ noted in [1], pp. 176):

Let

$$
\varphi: D \rightarrow \mathbb{N}^{k, 0} \subset \mathbb{C}^{k} \quad(D \subset \mathbb{C})
$$

be a holomorphic map. Let

$$
\begin{aligned}
X: D \rightarrow \mathbb{R}^{m, n} & (m+n=k) \\
X(\zeta) & =\left\{\begin{array}{l}
m\left\{\begin{array}{c}
\operatorname{Re} \\
\vdots \\
\operatorname{Re}
\end{array}\right. \\
n\{\varphi(\zeta) d \zeta . \\
\operatorname{Im} \\
\vdots \\
\operatorname{Im}
\end{array}\right.
\end{aligned}
$$

Then as long as

$$
\|\varphi\|_{m, n}^{2} \neq 0
$$

$X$ is an elliptic immersion, and is stationary. Thus $\varphi$ can be viewed as a mother who gives birth to a $2^{k}$-tuplet of stationary babies.

EXAMPLE 2.1. If $m=3, n=0$, then Theorem 2.1 reduces to the Enneper-Weierstrass representation for minimal surfaces in $\mathbb{R}^{3}$.

EXAMPLE 2.2. For $m=2, n=1, \mathrm{Gu}$ [1] developed an excellent geometric interpretation for stationary surfaces in 3-dimensional Minkowski space $\mathbb{R}^{2,1}$. His formula

$$
X(x, y)=\operatorname{Re} \int f(\zeta)(\cos \zeta, \sin \zeta, 1) d \zeta \quad(\zeta=x+i y)
$$

can be obtained from Theorem 2.1 by the following fact:

A holomorphic map $\varphi: D \rightarrow \mathbb{N}^{2,1}(D \subset \mathbb{C})$, with $\|\varphi\|_{2,1}^{2} \neq 0$, can always be locally written as

$$
\varphi(\zeta)=f(\zeta)(\cos \zeta, \sin \zeta, 1)
$$

except when $\varphi(D)$ lies in a complex line. 
EXAMPLE 2.3. $m=3, n=1$. Up to an isometry of $\mathbb{R}^{3,1}$, every elliptic stationary surface in $\mathbb{R}^{3,1}$ can locally be expressed by

$$
\begin{array}{r}
X(x, y)=\operatorname{Re} \int\left(4 f g h, 2 f g\left(1-h^{2}\right), f\left(1-g^{2}\right)\left(1+h^{2}\right),\right. \\
\left.f\left(1+g^{2}\right)\left(1+h^{2}\right)\right) d \zeta \\
(\zeta=x+i y)
\end{array}
$$

where $f(\zeta), g(\zeta)$ and $h(\zeta)$ are arbitrary holomorphic functions, and

$$
f g \neq 0, \quad(\operatorname{Im} g)^{2}+(\operatorname{Im} h)^{2} \neq 0 .
$$

Proof. Let $X$ be an elliptic stationary surface in $\mathbb{R}^{3,1}$. By Theorem 2.1 , locally there exists a holomorphic map

$$
\varphi: D \rightarrow \mathbb{N}^{3,1} \quad(D \subset \mathbb{C})
$$

such that

$$
\|\varphi\|_{3,1}^{2} \neq 0
$$

and

$$
X=\operatorname{Re} \int \varphi(\zeta) d \zeta
$$

Write $\varphi=\left(\varphi_{1}, \varphi_{2}, \varphi_{3}, \varphi_{4}\right)$; then

$$
\begin{aligned}
\varphi_{1}^{2}+\varphi_{2}^{2}+\varphi_{3}^{2}-\varphi_{4}^{2} & =0, \\
\left|\varphi_{1}\right|^{2}+\left|\varphi_{2}\right|^{2}+\left|\varphi_{3}\right|^{2}-\left|\varphi_{4}\right|^{2} & \neq 0 .
\end{aligned}
$$

Without the loss of generality, we may assume

$$
\varphi_{1} \neq 0 \text {. }
$$

Then, by a rotation in the $\left(x_{1}, x_{2}\right)$-plane, we may assume

$$
\varphi_{1} \neq 0, \quad \varphi_{1}^{2}+\varphi_{2}^{2} \neq 0 \text {. }
$$

Thus $\varphi_{3} \neq \pm \varphi_{4}$, and

$$
\begin{aligned}
& h=-\frac{1}{\varphi_{1}}\left(\varphi_{2}+\sqrt{\varphi_{1}^{2}+\varphi_{2}^{2}}\right), \\
& g=-\sqrt{\frac{\varphi_{4}-\varphi_{3}}{\varphi_{4}+\varphi_{3}}}, \\
& f=\frac{\varphi_{1}}{4 g h}
\end{aligned}
$$

are holomorphic functions. Now it is easy to see that

$$
\begin{aligned}
& \varphi_{1}=4 f g h, \quad \varphi_{2}=2 f g\left(1-h^{2}\right), \quad \varphi_{3}=f\left(1-g^{2}\right)\left(1+h^{2}\right), \\
& \varphi_{4}=f\left(1+g^{2}\right)\left(1+h^{2}\right) .
\end{aligned}
$$


EXAMPle 2.4. As in Example 2.3, up to a rigid motion, an elliptic stationary surface in $\mathbb{R}^{m, n}$ can be locally expressed by

$$
\begin{aligned}
X=\operatorname{Re} \int\left(2^{N-1}\right. & \prod_{j=1}^{N} f_{j}, \\
& 2^{N-k}\left(\prod_{j=1}^{N-k+1} f_{j}\right)\left(1-f_{N-k+2}^{2}\right) \\
& \times \prod_{j=N-k+3}^{N}\left(1+f_{j}^{2}\right),(k=2, \ldots, m), \\
& 2^{N-k} \sqrt{-1}\left(\prod_{j=1}^{N-k+1} f_{j}\right)\left(1-f_{N-k+2}^{2}\right) \\
& \times \prod_{j=N-k+3}^{N}\left(1+f_{j}^{2}\right), \quad(k=m+1, \ldots, N), \\
& \left.\quad f_{1} \prod_{j=2}^{N}\left(1+f_{j}^{2}\right)\right) d \zeta \\
&
\end{aligned}
$$

where $f_{j}, j=1, \ldots, N$, are holomorphic functions of $\zeta$.

B. Hyperbolic stationary surfaces.

THEOREM 2.2. Suppose $X: S \rightarrow \mathbb{R}^{m, n}$ is a hyperbolic stationary surface, and $p \in S$. Then there exists a neighborhood $U$ of $p$, and characteristic coordinates $(x, y)$ on $U$, such that $X$ can be written

$$
X(x, y)=X(0)+\int_{0}^{x} Y(x) d x+\int_{0}^{y} Z(y) d y
$$

where

$$
Y, Z:(-\varepsilon, \varepsilon) \rightarrow K^{m, n}
$$

are smooth (assume $(-\varepsilon, \varepsilon) \times(-\varepsilon, \varepsilon) \subset U)$, satisfying

$$
\langle Y(x), Z(y)\rangle \neq 0 \text { for all }(x, y) \text {. }
$$

Conversely, any smooth maps (6) satisfying (7) give rise to a hyperbolic stationary surface (5) in $\mathbb{R}^{m, n}$. 
Proof. Suppose $X: S \rightarrow \mathbb{R}^{m, n}$ is a hyperbolic immersion. Then under the characteristic coordinates $(x, y)$ on $S$, the map $X$ is stationary iff

$$
X_{x y}=0
$$

Thus

$$
Y=X_{x}, \quad Z=X_{y}
$$

are one-variable functions, and

$$
\begin{aligned}
X(x, y) & =X(0,0)+\int_{0}^{x} X_{x}(x, 0) d x+\int_{0}^{y} X_{y}(x, y) d y \\
& =X(0)+\int_{0}^{x} Y(x) d x+\int_{0}^{y} Z(y) d y .
\end{aligned}
$$

This proves formula (5). Since $(x, y)$ are characteristic coordinates, the images of $Y$ and $Z$ lie in $K^{m, n}$. Since $X$ is an immersion, (7) holds. Conversely, it is straightforward to derive (8) from (5), (6) and (7).

Theorems 2.1 and 2.2 can be unified into the following version:

In the elliptic case, let $\varphi: D \rightarrow \mathbb{N}^{m, n}(D \subset \mathbb{C})$ be holomorphic and $\|\varphi\|_{m, n}^{2} \neq 0$; in the hyperbolic case, let $\varphi(\zeta)=Y(x)-i Z(y): \mathbb{C} \rightarrow$ $K+i K\left(K=K^{m, n}\right)$ be smooth and $\langle\varphi, \varphi\rangle_{m, n}=2\langle Y, Z\rangle_{m, n} \neq 0$. In both cases, let

$$
X=\operatorname{Re} \int_{\zeta_{0}}^{\zeta} \varphi(\zeta) d \zeta \quad(\zeta=x+i y)
$$

which does not depend on the route from $\zeta_{0}$ to $\zeta$. Then

$$
X: D \rightarrow \mathbb{R}^{m, n}
$$

is a stationary immersion. In elliptic case, $(x, y)$ are the isothermal coordinates; in hyperbolic case, $(x, y)$ are the characteristic coordinates. Moreover, every elliptic or hyperbolic stationary surface comes from this manner.

This statement shows that the stationary surface is heavily related to the null cone.

EXAMPLE 2.5. Since every regular smooth map $F: R \rightarrow K^{2,1}$ can locally be written as

$$
F(s)=s(\cos \lambda, \sin \lambda, 1)
$$


where $\lambda=\lambda(s)$ is a smooth function, a hyperbolic stationary surface in $\mathbb{R}^{2,1}$ can locally be expressed by

$$
X=\int s(\cos \lambda, \sin \lambda, 1) d s+t(\cos \mu, \sin \mu, 1) d t
$$

where $\lambda=\lambda(s)$ and $\mu=(t)$ are smooth functions, and $\cos (\lambda-\mu) \neq 1$. If

$$
\frac{d \lambda}{d s} \neq 0, \quad \frac{d \mu}{d t} \neq 0,
$$

then we can use $(\lambda, \mu)$ as local coordinates, and write the surface as

$$
X=\int f(\lambda)(\cos \lambda, \sin \lambda, 1) d \lambda+g(\mu)(\cos \mu, \sin \mu, 1) d \mu .
$$

This is Gu's formula ([1]).

EXAMPLE 2.6. Every hyperbolic stationary surface in $\mathbb{R}^{3,1}$ can locally be expressed by

$$
\begin{aligned}
X=\int s & (\cos \alpha \cos \beta, \cos \alpha \sin \beta, \sin \alpha, 1) d s \\
& +t(\cos \lambda \cos \mu, \cos \lambda \sin \mu, \sin \lambda, 1) d t
\end{aligned}
$$

where $\alpha(s), \beta(s), \lambda(t), \mu(t)$ are smooth functions, satisfying

$$
\sin \alpha \sin \lambda \neq 1, \quad \text { and } \quad\{\cos (\beta-\mu) \neq \pm 1 \text { or } \cos (\alpha \mp \lambda) \neq 1\} \text {. }
$$

There is a similar formula for hyperbolic stationary surfaces in $\mathbb{R}^{m, n}$.

EXAMPLE 2.7. There is another representation formula for hyperbolic stationary surfaces. By the same method as in Example 2.3, one can prove that a hyperbolic stationary surface in $\mathbb{R}^{3,1}$, up to a rigid motion, can locally be written

$$
\begin{gathered}
X=\int\left(4 f_{1} f_{2} f_{3}, 2 f_{1} f_{2}\left(1-f_{3}^{2}\right), f_{1}\left(1-f_{2}^{2}\right)\left(1+f_{3}^{2}\right)\right. \\
\left.f_{1}\left(1+f_{2}^{2}\right)\left(1+f_{3}^{2}\right)\right) d \lambda \\
+\left(4 g_{1} g_{2} g_{3}, 2 g_{1} g_{2}\left(1-g_{3}^{2}\right), g_{1}\left(1-g_{2}^{2}\right)\left(1+g_{3}^{2}\right)\right. \\
\left.g_{1}\left(1+g_{2}^{2}\right)\left(1+g_{3}^{2}\right)\right) d \mu
\end{gathered}
$$

where $f_{j}(\lambda), g_{j}(\mu), j=1,2,3$, are smooth functions satisfying certain nondegenerate conditions. There is a similar expression for hyperbolic stationary surfaces in $\mathbb{R}^{m, n}$. 


\section{A construction of the stationary surface of mixed type.}

LemmA 3.1. Let $X: S \rightarrow \mathbb{R}^{m, n}$ be a stationary surface. Suppose $\gamma$ is a differentiable curve in $X$, consisting of parabolic points only. Then $\gamma$ is a null curve. In particular, the borderline in a mixed stationary surface is a null curve.

Proof. Suppose at some point $p \in \gamma$,

$$
\langle\dot{\gamma}(p), \dot{\gamma}(p)\rangle \neq 0 \text {. }
$$

Then there exists a local frame $\left\{e_{1}, e_{2}\right\}$ in a neighborhood of $p$ on the surface, such that

$$
\begin{aligned}
& \left\langle e_{1}, e_{2}\right\rangle=0 \text { everywhere, } \\
& e_{1} \text { is tangent to } \gamma \text { on } \gamma .
\end{aligned}
$$

Then by integration, we can find a set of local coordinates $(u, v)$ in a neighborhood of $p$, such that, the metric can be written

$$
d s^{2}=E d u^{2}+G d v^{2}
$$

and the curve $\gamma$ is

$$
v=0 \text {. }
$$

We have the following information

$$
E \neq 0, \quad G=0 \text { on } \gamma, \quad F=\left\langle X_{u}, X_{v}\right\rangle=0 .
$$

Denote

$$
\begin{aligned}
& G=v^{n} f \quad(n \geq 1, f \neq 0), \\
& g=E G-F^{2}=E G .
\end{aligned}
$$

Since $X$ is stationary, $\Delta X=0$ (away from the $\gamma$ ). Since $F=0$,

$$
\begin{aligned}
0= & \Delta X=\frac{1}{E} X_{u u}+\frac{1}{G} X_{v v}+\frac{1}{2 E}\left(\ln \frac{G}{E}\right)_{u} X_{u}+\frac{1}{2 G}\left(\ln \frac{E}{G}\right)_{v} X_{v} \\
= & \frac{1}{E} X_{u u}+\frac{1}{2 E}\left(\ln \frac{f}{E}\right)_{u} X_{u}+v^{-n}\left(\frac{1}{f} X_{v v}+\frac{1}{2 f}\left(\ln \frac{E}{f}\right)_{v} X_{v}\right) \\
& -\frac{n}{2 f} v^{-(n+1)} X_{v} .
\end{aligned}
$$

Multiplying the above by $v^{n+1}$, letting $v \rightarrow 0$, we get a contradiction.

For any analytic surface, once we know a piece of it, we know all of it. Now from a regular analytic null curve $\gamma(\theta)$ in $\mathbb{R}^{m, n}$, we construct an analytic stationary surface of mixed type as follows: 
The hyperbolic part is

$$
X(\theta, \sigma)=\frac{1}{2}(\gamma(\theta+\sigma)+\gamma(\theta-\sigma))
$$

The elliptic part is

$$
Y(\theta, \sigma)=\frac{1}{2}(\gamma(\theta+i \sigma)+\gamma(\theta-i \sigma)) .
$$

If an analytic curve $\gamma$ satisfies

$$
\langle\dot{\gamma}(s), \dot{\gamma}(t)\rangle=0 \text { for all } s, t
$$

then up to an isometry of $\mathbb{R}^{m, n}, \gamma$ can be written as

$$
\gamma(t)=\sum_{j=1}^{\min (m, n)} f_{j}(t)(0, \ldots, 0,1,0, \ldots, 0, \stackrel{m+n-j}{1}, 0, \ldots)
$$

where $f_{j}$, for all $j$, are analytic functions. The "generated surface" $X \cup \gamma \cup Y$ either degenerates to $\gamma$, or becomes a surface with the induced metric form identically vanishing. Therefore we must exclude this case. In $\mathbb{R}^{n, 1}$ or $\mathbb{R}^{1, n}$, a curve satisfying (12) is a null straight line.

LEMMA 3.2. Let $\gamma$ be an analytic null curve in $\mathbb{R}^{m, n}$, satisfying

$$
\langle\dot{\gamma}(s), \dot{\gamma}(t)\rangle \neq 0 \text { for all } 0<s-t<\varepsilon
$$

for some fuxed $\varepsilon>0$. Then in a neighborhood of $\gamma$, the point set $X \cup \gamma \cup Y$, obtained from (10) and (11), is an analytic stationary surface in $\mathbb{R}^{m, n}$ of mixed type.

Proof. For small $\sigma>0$, from (10) and (13), we have

$$
\left\langle X_{\theta}, X_{\sigma}\right\rangle=\frac{1}{2}\langle\dot{\gamma}(\theta+\sigma), \dot{\gamma}(\theta-\sigma)\rangle \neq 0 \text {. }
$$

But

$$
\left\langle X_{\theta}, X_{\theta}\right\rangle=\left\langle X_{\sigma}, X_{\sigma}\right\rangle=0 .
$$

Therefore $X$ is an analytic surface. For the same reason, $Y$ is an analytic surface, too. It is easy to see that

$$
\lim _{\sigma \rightarrow 0} X(\theta, \sigma)=\lim _{\sigma \rightarrow 0} Y(\theta, \sigma)=\gamma(\theta) .
$$

Therefore $X \cup \gamma \cup Y$ is first a continuous surface in a neighborhood of $\gamma$. After applying a rotation in the ambient space $R^{m+n}$, the hyperbolic part $X$ can be locally written as a graph

$$
z=f(x, y) \quad\left(z=\left(z_{1}, \ldots, z_{m+n-2}\right)\right)
$$


where $f$ is a vector-valued analytic function. It falls into an identity if we plug in

$$
x=x_{1}(\theta, \sigma), \quad y=x_{2}(\theta, \sigma), \quad z=\left(x_{3}(\theta, \sigma), \ldots, x_{m+n}(\theta, \sigma)\right)
$$

by (10). Since everything is analytic, it also holds for

$$
\begin{aligned}
& x=x_{1}(\theta, i \sigma), \quad y=x_{2}(\theta, i \sigma), \\
& z=\left(x_{3}(\theta, i \sigma), \ldots, x_{m+n}(\theta, i \sigma)\right),
\end{aligned}
$$

i.e., the elliptic part $Y$ can also be expressed as the same graph (14). Thus we verified that $X \cup \gamma \cup Y$ is an analytic surface. Finally, the induced metric is

$$
d s^{2}= \begin{cases}\frac{1}{2}\langle\dot{\gamma}(\theta+i \sigma), \dot{\gamma}(\theta-i \sigma)\rangle\left(d \theta^{2}+d \sigma^{2}\right) & \text { (on elliptic part), } \\ \frac{1}{2}\langle\dot{\gamma}(\theta+\sigma), \dot{\gamma}(\theta-\sigma)\rangle\left(d \theta^{2}-d \sigma^{2}\right) & \text { (on hyperbolic part) } .\end{cases}
$$

Therefore

$$
\Delta X=0, \quad \Delta Y=0 .
$$

Thus $X \cup \gamma \cup Y$ is stationary in $\mathbb{R}^{m, n}$.

Combining these two lemmas, we get

THEOREM 3.3. In a Minkowski space $\mathbb{R}^{m, n}$, every analytic null curve $\gamma$ satisfying (13) generates an analytic mixed stationary surface through (10) and (11). Conversely, every analytic mixed stationary surface is generated by such a null curve.

EXAMPLE 3.1. In $\mathbb{R}^{n, 1}$ or $\mathbb{R}^{1, n}$, every non-straight analytic null curve generates an analytic mixed stationary surface. Let $(x(\theta), y(\theta))$ be a regular analytic plane curve (not a straight line). Set

$$
\gamma(\theta)=\left(x(\theta), y(\theta), \int \sqrt{\dot{x}(\theta)^{2}+\dot{y}(\theta)^{2}} d \theta\right) .
$$

Then $\gamma$ is a non-straight analytic null curve in $\mathbb{R}^{2,1}$, from which one can construct a mixed stationary surface in $\mathbb{R}^{2,1}$ by (10) and (11). This generalizes Gu's construction in [2], where an extra condition that the plane curve has positive curvature is required.

EXAMPLE 3.2. $\gamma(\theta)=(\cos \theta, \sin \theta, \operatorname{ch} \theta, \operatorname{sh} \theta)$ in $\mathbb{R}^{3,1}$ generates the mixed stationary surface

$$
\left\{\begin{array}{l}
\tan ^{-1} \frac{x_{2}}{x_{1}}=\operatorname{th}^{-1} \frac{x_{4}}{x_{3}} \\
\left\{\begin{array}{l}
\operatorname{ch}^{-1} \sqrt{x_{1}^{2}+x_{2}^{2}}=\cos ^{-1} \sqrt{x_{3}^{2}-x_{4}^{2}} \quad \text { (elliptic part) } \\
\text { or } \\
\cos ^{-1} \sqrt{x_{1}^{2}+x_{2}^{2}}=\operatorname{ch}^{-1} \sqrt{x_{3}^{2}-x_{4}^{2}} \quad \text { (hyperbolic part) }
\end{array}\right.
\end{array}\right.
$$


EXAMPLE 3.3. $\gamma(\theta)=\left(\frac{1}{a} \cos (a \theta), \frac{1}{a} \sin (a \theta), \cos \theta, \sin \theta\right)$ in $\mathbb{R}^{2,2}$ generates the mixed stationary surface

$$
\left\{\begin{array}{l}
\tan ^{-1} \frac{x_{2}}{x_{1}}=a \tan ^{-1} \frac{x_{4}}{x_{3}}, \\
\operatorname{ch}^{-1}\left(a \sqrt{x_{1}^{2}+x_{2}^{2}}\right)=a \operatorname{ch}^{-1} \sqrt{x_{3}^{2}+x_{4}^{2}} \quad \text { (elliptic part), } \\
\text { or } \\
\cos ^{-1}\left(a \sqrt{x_{1}^{2}+x_{2}^{2}}\right)=a \cos ^{-1} \sqrt{x_{3}^{2}+x_{4}^{2}} \quad \text { (hyperbolic part) } .
\end{array}\right.
$$

It is elliptic on $x_{3}^{2}+x_{4}^{2}>1$, hyperbolic on $x_{3}^{2}+x_{4}^{2}<1$.

In particular, if $a=2$, this is the mixed stationary surface

$$
\left\{\begin{array}{l}
x_{2}\left(x_{3}^{2}-x_{4}^{2}\right)=2 x_{1} x_{3} x_{4} \\
\sqrt{x_{1}^{2}+x_{2}^{2}}=x_{3}^{2}+x_{4}^{2}-\frac{1}{2} .
\end{array}\right.
$$

If $a=3$, this is the mixed stationary surface

$$
\left\{\begin{array}{l}
x_{2} x_{3}\left(x_{3}^{2}-3 x_{4}^{2}\right)=x_{1} x_{4}\left(3 x_{3}^{2}-x_{4}^{2}\right) \\
3 \sqrt{x_{1}^{2}+x_{2}^{2}}=\sqrt{x_{3}^{2}+x_{4}^{2}}\left(4\left(x_{3}^{2}+x_{4}^{2}\right)-3\right) .
\end{array}\right.
$$

EXAMPLE 3.4. $\gamma(\theta)=\left(\cos \theta, \sin \theta, \frac{1}{2} \cos 2 \theta, \frac{1}{2} \sin 2 \theta, \sqrt{2} \theta\right)$ in $\mathbb{R}^{4,1}$ generates the mixed stationary surface

$$
\begin{aligned}
& x_{3}=\frac{1}{2}\left(x_{1}^{2}-x_{2}^{2}\right)\left(2-\frac{1}{x_{1}^{2}+x_{2}^{2}}\right), \\
& x_{4}=x_{1} x_{2}\left(2-\frac{1}{x_{1}^{2}+x_{2}^{2}}\right), \\
& x_{5}=\sqrt{2} \arctan \frac{x_{2}}{x_{1}} .
\end{aligned}
$$

It is elliptic on $x_{1}^{2}+x_{2}^{2}>1$, hyperbolic on $x_{1}^{2}+x_{2}^{2}<1$.

\section{REFERENCES}

[1] C. $\mathrm{H} . \mathrm{Gu}$, The extremal surface in the 3-dimensional Minkowski space, Acta Math. Sinica, New Series, 1 (1985), 173-180.

[2] _ A Global Study of Extremal Surfaces in 3-Dimensional Minkowski Space, Lecture Notes in Math., vol. 1255, Springer-Verlag, Berlin and New York, pp. 26-33.

[3] R. Osserman, A survey of Minimal Surfaces, Van Nostrand, New York, 1969.

Received June 31, 1991. 



\title{
PACIFIC JOURNAL OF MATHEMATICS \\ Founded by \\ E. F. BECKENBACH (1906-1982) F. WolF (1904-1989)
}

\section{EDITORS}

\section{S. VARADARAJAN \\ (Managing Editor) \\ University of California \\ Los Angeles, CA 90024-1555 \\ vsv@math.ucla.edu}

\section{F. Michael Christ}

University of California

Los Angeles, CA 90024-1555

christ@math.ucla.edu

\section{Herbert Clemens}

University of Utah

Salt Lake City, UT 84112

clemens@math.utah.edu

\author{
THOMAs ENRIGHT \\ University of California, San Diego \\ La Jolla, CA 92093 \\ tenright@ucsd.edu \\ Nicholas ERcolani \\ University of Arizona \\ Tucson, AZ 85721 \\ ercolani@math.arizona.edu \\ R. FINN \\ Stanford University \\ Stanford, CA 94305 \\ finn@gauss.stanford.edu \\ VAUghan F. R. JoNeS \\ University of California \\ Berkeley, CA 94720 \\ vfr@math.berkeley.edu
}

\section{SUPPORTING INSTITUTIONS}

UNIVERSITY OF ARIZONA

UNIVERSITY OF BRITISH COLUMBIA

CALIFORNIA INSTITUTE OF TECHNOLOGY

UNIVERSITY OF CALIFORNIA

UNIVERSITY OF MONTANA

UNIVERSITY OF NEVADA, RENO

NEW MEXICO STATE UNIVERSITY OREGON STATE UNIVERSITY

\author{
UNIVERSITY OF OREGON \\ UNIVERSITY OF SOUTHERN CALIFORNIA \\ STANFORD UNIVERSITY \\ UNIVERSITY OF HAWAII \\ UNIVERSITY OF UTAH \\ WASHINGTON STATE UNIVERSITY \\ UNIVERSITY OF WASHINGTON
}

The Supporting Institutions listed above contribute to the cost of publication of this Journal, but they are not owners or publishers and have no responsibility for its content or policies.

Mathematical papers intended for publication in the Pacific Journal of Mathematics should be in typed form or offset-reproduced (not dittoed), double spaced with large margins. Please do not use built up fractions in the text of the manuscript. However, you may use them in the displayed equations. Underline Greek letters in red, German in green, and script in blue. The first paragraph must be capable of being used separately as a synopsis of the entire paper. In particular it should contain no bibliographic references. Please propose a heading for the odd numbered pages of less than 35 characters. Manuscripts, in triplicate, may be sent to any one of the editors. Please classify according to the 1991 Mathematics Subject Classification scheme which can be found in the December index volumes of Mathematical Reviews. Supply name and address of author to whom proofs should be sent. All other communications should be addressed to the managing editor, or Julie Speckart, University of California, Los Angeles, California 90024-1555.

There are page-charges associated with articles appearing in the Pacific Journal of Mathematics. These charges are expected to be paid by the author's University, Government Agency or Company. If the author or authors do not have access to such Institutional support these charges are waived. Single authors will receive 75 free reprints; joint authors will receive a total of 100 free reprints. Additional copies may be obtained at cost in multiples of 50 .

The Pacific Journal of Mathematics (ISSN 0030-8730) is published monthly except for July and August. Regular subscription rate: $\$ 200.00$ a year (10 issues). Special rate: $\$ 100.00$ a year to individual members of supporting institutions.

Subscriptions, orders for numbers issued in the last three calendar years, and changes of address should be sent to Pacific Journal of Mathematics, P.O. Box 969, Carmel Valley, CA 93924, U.S.A. Old back numbers obtainable from Kraus Periodicals Co., Route 100, Millwood, NY 10546.

The Pacific Journal of Mathematics at P.O. Box 969, Carmel Valley, CA 93924 (ISSN 0030-8730) is published monthly except for July and August. Second-class postage paid at Carmel Valley, California 93924, and additional mailing offices. Postmaster: send address changes to Pacific Journal of Mathematics, P.O. Box 969, Carmel Valley, CA 93924.

PUBLISHED BY PACIFIC JOURNAL OF MATHEMATICS, A NON-PROFIT CORPORATION

This publication was typeset using $\mathcal{A} \mathcal{S} S-\mathrm{T}_{\mathrm{E}} \mathrm{X}$, the American Mathematical Society's $\mathrm{T}_{\mathrm{E}} \mathrm{X}$ macro system.

Copyright (c) 1993 by Pacific Journal of Mathematics 


\section{PACIFIC JOURNAL OF MATHEMATICS}

Volume $158 \quad$ No. $2 \quad$ April 1993

On the extension of Lipschitz functions from boundaries of subvarieties to

201 strongly pseudoconvex domains

K. ADACHI and HiRoshi KAJIMOTO

On a nonlinear equation related to the geometry of the diffeomorphism group 223

DaVid DaI-WAi BaO, JACQUES LAFONTAINE and TUdOR S. RATIU

Fixed points of boundary-preserving maps of surfaces

ROBERT F. BROWN and BRIAN SANDERSON

On orthomorphisms between von Neumann preduals and a problem of Araki 265

L. J. BUNCE and JOHN DAVID MAITLAND WRIGHT

Primitive subalgebras of complex Lie algebras. I. Primitive subalgebras of 273 the classical complex Lie algebras

\section{V. CHEKALOV}

$L^{n}$ solutions of the stationary and nonstationary Navier-Stokes equations in 293 $R^{n}$

\section{ZHI MiN CHEN}

Some applications of Bell's theorem to weakly pseudoconvex domains

XiAO JUN HUANG

On isotropic submanifolds and evolution of quasicaustics

STANISŁAW JANECZKO

Currents, metrics and Moishezon manifolds

SHANYU JI

Stationary surfaces in Minkowski spaces. I. A representation formula

JIANGFAN LI

The dual pair $(U(1), U(1))$ over a $p$-adic field

Courtney Hughes Moen

Any knot complement covers at most one knot complement 